ТЕРМОДИНАМИЧЕСКИЕ

ОСНОВЫ АЭЭ

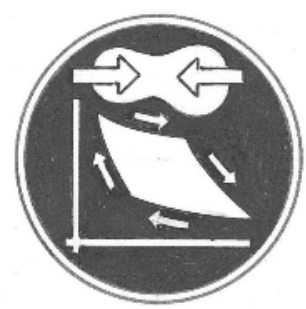

THERMODYNAMIC

BASICS OF AEE

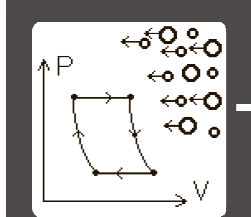

\title{
ТЕРМОДИНАМИЧЕСКИЙ АНАЛИЗ
}

\section{THERMODYNAMIC ANALYSIS}

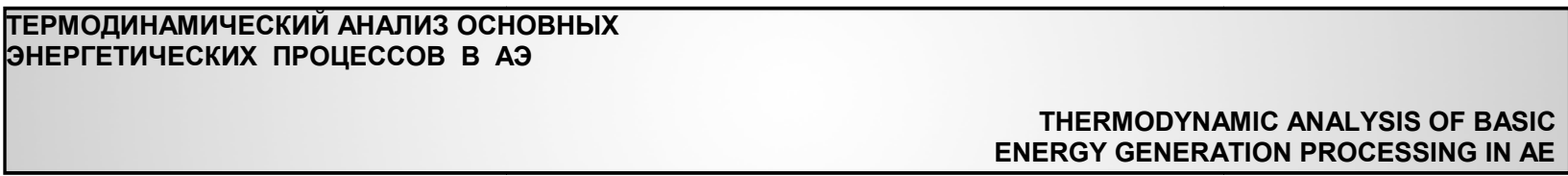

Статья поступила в редакцию 27.03.15. Ред. рег. № 2208

The article has entered in publishing office 27.03.15. Ed. reg. No. 2208

\section{УДК 66.047.75.021.4 \\ МОДЕЛЬ ТЕПЛОМАССОПЕРЕНОСА В ПЕЧАХ ПРИ ПРОИЗВОДСТВЕ КАРБИДА КРЕМНИЯ}

\author{
В.С. Кузеванов, Г.С. Закожсурникова, С.С. Закожурников \\ Филиал ФГБОУ ВПО Национальный исследовательский университет «МЭИ» \\ РФ 404110, Волгоградская обл., г. Волжский, пр. Ленина, 69 \\ тел.: (8443)21-01-60; факс: (8443)21-01-66; e-mail: vfmei@vfmei.ru \\ galya.vlz@mail.ru
}

doi: 10.15518/isjaee.2015.07.006

Заключение совета рецензентов: 03.04.15 Заключение совета экспертов: 10.04.15 Принято к публикации: 20.04.15

Предметом исследования является процесс тепломассообмена в печах сопротивления при производстве карбида кремния. В общей физической модели процесса рассматривается твёрдое тело с газовыми полостями и внутренним тепловыделением. Твёрдая составляющая многокомпонентна и увлажнена. Учитываются теплопроводность при переменных теплофизических параметрах, переменная пористость, тепло химических реакций, процесс сушки твёрдой составляющей, генерация газовой составляющей в химических реакциях и перенос тепла фильтрационным потоком газа.

Математическое описание процесса представляет собой систему из двух дифференциальных уравнений: уравнения теплопроводности для пористого тела с химически реагирующими компонентами и уравнения переноса тепла в процессе фильтрации газа через взаимосвязанные поры. Дифференциальные уравнения дополнены необходимыми замыкающими соотношениями.

Замыкающие соотношения получены в результате рассмотрения моделей процессов, сопутствующих производству карбида кремния: сушки, расчёта поля давления в системе связанных пор, удельного тепла химических реакций и эффективного коэффициента теплопроводности.

Полученная математическая модель позволяет определить температурные поля, подобрать варианты загрузок печи и режимов плавки при производстве карбида кремния.

Ключевые слова: пористый материал, производство карбида кремния, физическая модель, математическая модель, температурные поля, тепломассоперенос, объемные тепловыделения.

International Scientific Journal for Alternative Energy and Ecology

(C) Scientific Technical Centre «TATA», 2015

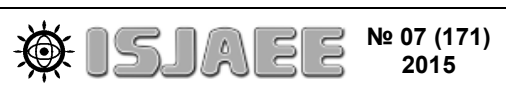

75 


\title{
HEAT AND MASS TRANSFER MODEL IN FURNACES IN THE SILICON CARBIDE PRODUCTION
}

\author{
V.S. Kuzevanov, G.S. Zakozhurnikova, S.S. Zakozhurnikov \\ Volzhsky Branch of the National Research University "Moscow Power Engineering Institute" \\ 69 Lenin Ave., Volzhsky, Volgograd reg., 404110 Russian Federation \\ ph.: (8443)21-01-60; fax: (8443)21-01-66; e-mail: vfmei@vfmei.ru \\ galya.vlz@mail.ru
}

Referred 03 April 2015 Received in revised form 10 April 2015 Accepted 20 April 2015

The paper researches the process of heat and mass transfer in furnaces of the resistance in the silicon carbide production and considers a solid body with gas cavities and internal heat in general physical model of the process. Solid part is multicomponent and moist. The thermal conductivity with variable thermal parameters, variable porosity, the heat of chemical reactions, the process of drying the solid component, the generation of gas in chemical reactions and heat transfer seepage gas flow are taking into account.

The mathematical description of the process is a system of two differential equations: the heat equation for a porous body with a chemically reactive components and heat transfer equation in the process of filtering the gas through the interconnected pores. Differential equations are supplemented by the necessary closing relations.

Closing relations are the results of the considering of the processes model accompanying the silicon carbide production: the drying, calculating the pressure field in the system of interconnected pores, heat of chemical reactions and the effective thermal conductivity.

The obtained mathematical model allows determining the temperature field, choosing variants of loading the furnace and regimes of melting in the silicon carbide production.

Keywords: porous material, production of silicon carbide, physical model, mathematical model, temperature fields, heat and mass transfer, volumetric heat generation.

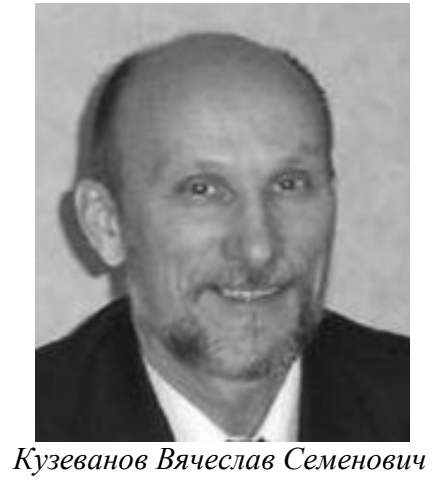

Vyacheslav S. Kuzevanov

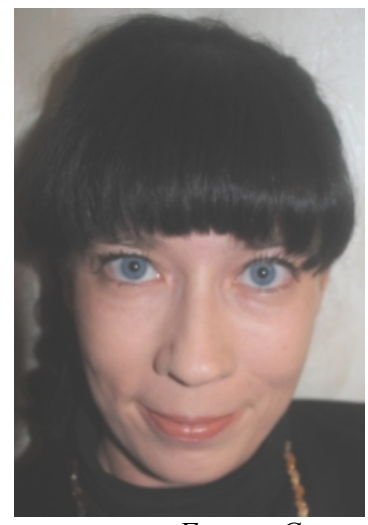

Закожурникова Галина Сергеевна Galina S. Zakozhurnikova
Сведения об авторе: д-р техн. наук, профессор кафедры «Теплоэнергетика и теплотехника» филиала ФГБОУ ВПО «Национальный исследовательский университет «МЭИ» в г. Волжском.

Образование: Московский энергетический институт.

Область научных интересов: тепловая и атомная энергетика, ресурсосбережение, моделирование технологических процессов.

Публикации: 142.

Сведения об авторе: старший преподаватель кафедры «Промышленная теплоэнергетика» филиала ФГБОУ ВПО «Национальный исследовательский университет «МЭИ» в г. Волжском.

Образование: Московский энергетический институт (технический университет).

Область научных интересов: тепломассообмен.

Публикации: 14.
Information about the author: DSc (tech.), professor of a chair of Thermal Engineering and Heat Engineering Department Volzhsky Branch of the National Research University "Moscow Power Engineering Institute".

Education: Moscow Power Engineering Institute.

Research area: thermal and nuclear energy, resource conservation, modeling of technological processes.

Publications: 142.

Information about the author: senior lecture of a chair of Industrial Thermal Power Engineering Department of Volzhsky Branch of the National Research University "Moscow Power Engineering Institute".

Education: Moscow Power Engineering Institute.

Research area: heat and mass transfer.

Publications: 14 . 


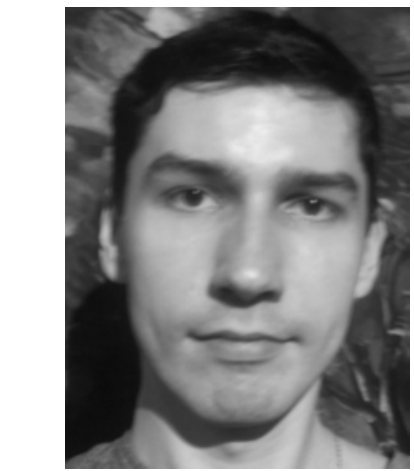

Закожурников Сергей Сергеевич Sergei S. Zakozhurnikov
Сведения об авторе: аспирант кафедры «Тепломассообменных процессов и установок» ФГБОУ ВПО «Национальный исследовательский университет «МЭИ», Москва

Образование: Московский энергетический институт (технический университет).

Область научных интересов: моделирование технологических процессов, ресурсосбережение, энергосбережение.

Публикации: 18.
Information about the author: postgraduate student of National Research University "Moscow Power Engineering Institue".

Education: Moscow Power Engineering Institute.

Research area: modeling of technological processes, resource conservation, energy conservation.

Publications: 18 .

\section{Введение}

Предметом исследования является процесс тепломассообмена в печах сопротивления при производстве карбида кремния. Карбид кремния ( $\mathrm{SiC})$ используется во многих отраслях промышленности: огнеупорной, металлургической, керамической, электротехнической. $\mathrm{SiC}$ обладает рядом полезных свойств, таких как высокая прочность, низкий коэффициент теплового расширения, высокая электрическая проводимость, нелинейное электрическое сопротивление. Карбид кремния является материалом, попадающим в диапазон твёрдости между алмазом и корундом (между 9,5 и 10 по шкале Мооса). Он достаточно стабилен химически и устойчив к действию кислот. Благодаря высокой твёрдости, карбид кремния является основным сырьём для изготовления абразивных труб, крыльчаток, насосных камер и т. д. Большая зернистость карбида кремния используется при создании таких абразивных изделий, как наждачная бумага, шлифовальные круги, диски, проволочные пилы и ряда других. Также карбид кремния применяют в технологиях хранения радиоактивных отходов.

На сегодняшний день производство $\mathrm{SiC}$ остаётся во многом таким же, как и в конце XIX века: кварц высокой чистоты смешивают с высококачественным коксом или антрацитом в больших электрических печах сопротивления, и при нагреве до температуры выше $2000{ }^{\circ} \mathrm{C}$ получают карбид кремния в соответствии со следующей реакцией: $\mathrm{SiO}_{2}+3 \mathrm{C}=\mathrm{SiC}+2 \mathrm{CO}$.

Процесс плавки сопровождается значительным теплоподводом, требуя 8 000-10 000 кВт·ч электрической энергии на тонну конечного продукта.

Карбид кремния извлекают из печи, охлаждают и затем сортируют на различные классы. Сортировка применяется для отделения полноценного кристаллического карбида (карборунда) от металлургического карбида кремния. Отсортированный карборунд затем измельчают и просеивают до нужного вида.

Образование $\mathrm{SiC}$ сопровождается и другими химическими реакциями, в результате которых образуются твердые и газообразные продукты. Теплота химических реакций, образование дополнительной газовой составляющей, процесс сушки, перенос тепла фильтрационным потоком газа оказывают значительное влияние на процесс тепломассообмена и в целом на процесс производства $\mathrm{SiC}$.

Экспериментальное исследование нестационарного тепломассообмена в печах для производства карбида кремния усложнено высокими температурами в процессе карбидообразования.

В таких объективно ограниченных условиях анализа важным является расчетное исследование плавки с целью определения режимов с повышенным выходом SiC. Основой исследования при этом является нахождение распределения температур в печи и определение его влияния на увеличение массового выхода продукта.

\section{Общая физическая модель процесса}

В работе рассматривается твердое тело (индекс «1») с газовыми полостями (индекс «2») и внутренним тепловыделением. Твердая составляющая многокомпонентна и увлажнена.

Предполагается, что перенос тепла за счет молекулярной теплопроводности может быть описан как для сплошной среды с теплофизическими параметрами, эквивалентными соответствующим параметрам пористого объекта.

\section{В модели учитывается:}

1. Теплопроводность при переменных теплофизических параметрах $(\lambda, \rho, c)$.

2. Переменная пористость $(\varepsilon)$.

3. Химические реакции:

- тепло химических реакций;

- образование дополнительной газовой составляющей.

4. Процесс сушки.

5. Перенос тепла фильтрационным потоком газа.

Значимые параметры и допущения:

1. Масса системы $(M)$.

Изменение массы связано с химическими реакциями и сушкой с образованием летучих продуктов плавки (выходом газа и пара (далее газ) из системы). 


\section{2. Температура $(T)$.}

Температура определена состоянием твёрдой пористой структуры. Температура газа равна температуре твёрдой структуры в соответствующей локальной области в любой момент времени.

3. Давление $(P)$.

Давление определено состоянием газовой составляющей.

4. Пористость $(\varepsilon)$.

Изменение пористости связано с изменением массы твёрдой составляющей, то есть с химическими реакциями.

5. Константы химических реакций $\left(k_{\mathrm{p}, i}\right)$ :

- $k_{\mathrm{p}, i}$ определяют скорость изменения состава и массы твёрдой структуры;

- $k_{\mathrm{p}, i}$ определяют скорость изменения состава и плотности массового расхода газовой составляющей.

6. Влажность твёрдой структуры $\left(x_{\mathrm{B}}\right)$.

Влага равномерно распределена в сухой твёрдой составляющей (твердых компонентах) и находится в микропорах, не связанных между собой каналами. Концентрация микропор в твердом компоненте, не участвующем в химической реакции, неизменна.

Изменение влажности определено процессом сушки. Сушка сопровождается изменением состава и плотности массового расхода газовой составляющей за счёт парового компонента, а также изменением массы составляющей, отнесённой к твёрдой.

\section{Базовые элементы модели переноса тепла:}

1. Уравнение теплопроводности в системе, изменяющейся вследствие химических реакций и сушки, является основным уравнением баланса энергии;

2. Учитывается перемещение газовой составляющей, сопровождающееся переносом тепла;

3. Характерные времена процессов «1» и «2» считаются таковыми, что перемещение газовой составляющей можно рассчитывать в квазистационарном приближении;

4. Состояние газовой составляющей описывается уравнением для смеси идеальных газов.

5. В процессе химических превращений и сушки изменяется пористость системы $\left(\varepsilon=\frac{\mathrm{v}_{2}}{\mathrm{v}}\right)$, но не меняется её произвольно выбранный объём v ;

6. Перепады давления в системе и уровень изменения давления малы. Поле давления определяет фильтрационные потоки, но при расчете теплофизических параметров процесс можно полагать изобарным.

Таким образом, совокупный тепломассоперенос представлен как нестационарный процесс теплопроводности, на который наложен процесс перераспределения тепла в пористом теле вследствие тепломассопереноса фильтрационным потоком газа.

\section{Математическая модель}

Для определения температурного поля в печи для производства $\mathrm{SiC}$ запишем дифференциальное уравнение энергии в виде, не учитывающем массоперенос:

$$
\frac{\partial p h}{\partial \tau}=-\operatorname{div} q+q_{\mathrm{v}}
$$

где $\rho$ - плотность; $h$ - энтальпия; $q_{\mathrm{v}}-$ мощность внутренних источников тепла в теле.

Заметим, что уравнение (1) является, по существу, уравнением теплопроводности для тела с переменными теплофизическими свойствами.

Определим $\rho h$ для конкретной пористой системы как:

$\rho h=\rho_{1}(1-\varepsilon) h_{1}+\rho_{2} \varepsilon h_{2}$.

Рассмотрим элементарную ячейку постоянного объема v, в которой согласно модели сплошной среды содержится и составляющая 1 , и составляющая 2 в пропорциях, определенных их объемными долями. Перепишем уравнение (2) для всех компонентов объема v:

$\rho h=\tilde{\rho}_{1}^{\mathrm{c}} \cdot h_{1}^{\mathrm{c}}+\tilde{\rho}_{\text {газ }} \cdot h_{\text {газ }}+\tilde{\rho}_{\text {в }} \cdot h_{\text {в }}+\tilde{\rho}_{\text {пар }} \cdot h_{\text {пар }}+\tilde{\rho}_{2} h_{2}$,

где верхний индекс « » относится к парциальной плотности $\tilde{\rho}_{i}$, определяемой как $\tilde{\rho}_{i}=\lim _{\mathrm{v} \rightarrow 0} \frac{m_{i}}{\mathrm{v}} ;$ где $m_{i}$ - масса компонента «i» в элементарной ячейке; $\tilde{\rho}_{1}$, $h_{1}^{\mathrm{c}}-$ парциальная плотность и энтальпия твердого сухого компонента; $\tilde{\rho}_{\text {газ }}, h_{\text {газ }}-$ парциальная плотность и энтальпия газа, появляющегося в результате химических реакций в твердом сухом компоненте; $h_{\mathrm{B}}, \tilde{\rho}_{\mathrm{в}}-$ энтальпия водяной фазы и парциальная плотность, определяющая влажность твердого компонента; $\tilde{\rho}_{\text {пар }}, h_{\text {пар }}-$ парциальная плотность и энтальпия пара, появляющегося из воды в результате фазового перехода при сушке твердого компонента; $\tilde{\rho}_{2}, h_{2}-$ парциальная плотность и энтальпия газовой составляющей в связанных порах.

Учтем, что в отсутствие массопереноса

$$
\left.\begin{array}{l}
\tilde{\rho}_{1}^{c}+\tilde{\rho}_{\text {газ }}+\tilde{\rho}_{1,0}^{c}=\text { const, } \\
\tilde{\rho}_{\text {в }}+\tilde{\rho}_{\text {пар }}+\tilde{\rho}_{\text {в,0 }}=\text { const }, \\
\tilde{\rho}_{2}+\tilde{\rho}_{2,0}=\text { const, }
\end{array}\right\}
$$


где нижний индекс «0» соответствует начальным условиям, определенным в момент включения электронагрева системы (начало режима плавки).
Тогда из (3) и (4) и в приближении $\frac{\partial h_{i}}{\partial \tau}=C_{i} \frac{\partial T}{\partial \tau}$ производная по времени функции $\rho h$ будет иметь вид:

$$
\begin{aligned}
& \frac{\partial}{\partial \tau}(\rho h)=\left\{\rho_{1}^{\mathrm{c}}(1-\varepsilon)(1+v)^{-1} C_{1}^{\mathrm{c}}+(1+v)^{-1}\left[\rho_{1,0}^{\mathrm{c}}\left(1-\varepsilon_{0}\right)-\rho_{1}^{\mathrm{c}}(1-\varepsilon)\right] C_{\rho, \text { газ }}+\rho_{\mathrm{в}} \frac{(1-\varepsilon)}{1+\nu} v C_{\mathrm{B}}+\frac{v}{1+\nu}\left[\rho_{\mathrm{B}, 0}\left(1-\varepsilon_{0}\right)-\rho_{\mathrm{B}}(1-\varepsilon)\right] C_{\rho, \text { пар }}+\right. \\
& \left.+\rho_{2} \varepsilon C_{\rho, 2}\right\} \times \frac{\partial T}{\partial \tau}+\frac{1}{1+v}\left(h_{1}^{\mathrm{c}}-h_{\text {газ }}\right) \frac{\partial\left[\rho_{1}^{\mathrm{c}}(1-\varepsilon)\right]}{\partial \tau}+\left(h_{\mathrm{в}}-h_{\text {пар }}\right) \cdot \frac{v}{1+v} \cdot \frac{\partial\left[\rho_{\mathrm{B}}(1-\varepsilon)\right]}{\partial \tau}
\end{aligned}
$$

Заметим, что в (5) правая часть записана через плотности $\rho_{i}$ и объемные доли каждого компонента, причем $v=\frac{\mathrm{v}_{\mathrm{B}}}{\mathrm{v}_{1}^{\mathrm{c}}}-$ доля не связанных между собой микропор - согласно принятой модели есть величина постоянная.

Обозначим интенсивность объемной генерации газа в химических реакциях как $\widetilde{g}_{\text {хим }}$ и пара в процессе сушки как $\tilde{g}_{\text {суш }}$. Очевидно, что $\tilde{g}_{\text {хим }}=-\frac{\partial \tilde{\rho}_{1}^{c}}{\partial \tau}=-\frac{1}{1+v} \frac{\partial\left[\rho_{1}^{\mathrm{c}}(1-\varepsilon)\right]}{\partial \tau}$,

a $\tilde{g}_{\text {суш }}=-\frac{\partial \tilde{\rho}_{\mathrm{B}}}{\partial \tau}=-\frac{v}{1+v} \frac{\partial\left[\rho_{\mathrm{в}}(1-\varepsilon)\right]}{\partial \tau}$.

Тогда, согласно физическому смыслу, две последние составляющие правой части соотношения (5) можно определить как внутренние источники (стоки) тепла, обусловленные процессами химических превращений $\left(q_{V, \text { хим }}\right)$ и сушкой твердого компонента $\left(q_{V \text {, суш }}\right)$ :

$\left.\begin{array}{l}q_{V, \text { хим }}=\left(h_{1}^{\mathrm{c}}-h_{\text {газ }}\right) \tilde{g}_{\text {хим }}=e \tilde{g}_{\text {хим }}, \\ q_{V, \text { суш }}=\left(h_{\text {пар }}-h_{\text {в }}\right) \tilde{g}_{\text {суш }}=r \tilde{g}_{\text {суш }},\end{array}\right\}$

где $e$ - теплота совокупности химических реакций, приводящих к появлению газовой составляющей; $r-$ скрытая теплота парообразования.

Перенесем эти составляющие удельного объемного тепловыделения в правую часть уравнения (1) при его записи с учетом полученных соотношений (5) и (6). Имеем:

$\Phi \frac{\partial T}{\partial t}=-\operatorname{div} q+q_{V, \text { эл }}+q_{V, \text { хим }}-q_{V, \text { суш }} \cdot$

Здесь Ф соответствует выражению в фигурных скобках правой части (5), а индекс «эл» относится к тепловыделению, обусловленному подводом электрической энергии.

Рассмотрим процесс переноса тепла, определяемый переносом массы вследствие перемещения (фильтрации) парогазовой составляющей в системе взаимосвязанных макропор. По аналогии с выражениями (6) можно записать:

$q_{V, \phi}=-\operatorname{div} q_{\phi}=e_{\phi} \tilde{g}_{\phi}$,

где индекс «ф» относится к процессу фильтрации.

Естественно, температурное поле в пористом теле зависит от $q_{V, \phi}$, но и $q_{V, \phi}$ зависит от температурного поля, поскольку температура определяет теплофизические параметры смеси газов, а совместно с характеристиками проницаемости обусловливает поле давления в газовой составляющей, и, следовательно, $\tilde{g}_{\phi}$ и $q_{V, \phi}$.

Наша общая модель физического процесса позволяет представить математическую модель как систему уравнений:

$\left.\begin{array}{l}\Phi \frac{\partial T}{\partial t}=-\operatorname{div} q+q_{V, \text { эл }}+q_{V, \text { хим }}-q_{V, \text { суш }}+q_{V, \phi}, \\ q_{V, \phi}=q_{V, \phi}\left\{T, \frac{\partial T}{\partial t}, \tilde{g}_{\text {хим }}, \tilde{g}_{\text {суш }}, P, \frac{\partial P}{\partial x_{i}}\right\} .\end{array}\right\}$

Модели расчета объемных тепловыделений $q_{V, i}$

\section{Определение $q_{V \text {,эл }}$.}

Принято, что базовая доля $q_{V \text {,эл }}^{\mathrm{K}}$ энерговыделения приходится на греющий элемент плавильной печи (керн). Поскольку электрическое сопротивление шихты зависит от влажности, а также от наличия $\mathrm{SiC}$ как составляющей с высокой электрической проводимостью, принято

$$
q_{V, \text { эл }}^{\mathrm{K}}=q_{V, \text { эл }}^{0} \frac{\left(1-\bar{x}_{\mathrm{B}}\right)\left(1-x_{\mathrm{SiC}}\right)}{1-x_{\mathrm{B}, 0}} .
$$


И в любой момент времени проверяется выполнение условия:

$Q_{\text {эл }}=\int_{V_{n}} q_{V, \text { эл }} d V$

В соотношениях (10), (11) индекс «0» соответствует началу плавки; $x_{\mathrm{SiC}}-$ массовая доля $\mathrm{SiC}$ в шихте; $\bar{x}_{\text {в }}$ - средняя влажность твёрдой составляющей шихты; $V_{n}$ - объём загрузки плавильной печи.

\section{определение $q_{V \text {, им }}$}

Удельное тепло всех «n» принятых во внимание химических реакций

$$
q_{V, \text { хим }}=\sum_{j=1}^{n} q_{V, j}=\sum_{j=1}^{n} e_{j} \tilde{g}_{\text {хим. } . j}
$$

где $e_{j}$ - тепло химической реакции «ј».

Общее удельное выделение летучих продуктов реакции «j» за время $\tau$

$$
G_{\text {хим }, j}=\int_{0}^{\tau} \tilde{g}_{\text {хим }, j} \delta_{i, j} d \tau \text {. }
$$

Здесь $\delta_{i, j}=\left\{\begin{array}{l}1, \text { если } \tau_{1, j}<\tau<\tau_{2, j}, \\ 0, \text { если } \tau \leq \tau_{1, j} \text { или } \tau \geq \tau_{2, j} .\end{array}\right.$

В условиях (13) по определению функции $\delta_{i, j}$ через $\tau_{1, j}$ и $\tau_{2, j}$ обозначены время начала и завершения химической реакции типа «j» соответственно.

Считаем, что $\widetilde{g}_{\text {хим,j }}$ есть функция, определяемая только видом химической реакции, и что сама реакция протекает при достижении температуры реагирующих компонентов значения $T_{\text {н.p. }}$ - температуры начала химической реакции типа «ј», а значение $g_{\text {хим, } j}$ при наличии всех компонентов реакции и условии $T(x, y, z)>T_{\text {н.p. } j}$ не зависит от координат $(x, y, z)$ и времени.

Тогда $G_{\text {хим }, j}=\tilde{g}_{\text {хим }, j} \int_{0}^{\tau} \delta_{i, j} d \tau$ и в целом по расчётной зоне $V_{\mathrm{p}, j}$, где сосредоточены соответствующие виду химической реакции компоненты,

$$
\begin{aligned}
& \widetilde{G}_{\text {хим }, j}=\widetilde{g}_{\text {хим }, j} \int_{V_{\mathrm{p}, j}} \int_{0}^{\tau} \delta_{i, j} d \tau d V \text { или } \\
& \widetilde{G}_{\text {хим }, j}=\widetilde{g}_{\text {хим }, j} \int_{V_{\mathrm{p}, j}}\left(\tau_{2, j}-\tau_{1, j}\right) d V,
\end{aligned}
$$

где $\tau_{1, j}$ - время достижения температуры начала химической реакции типа «j» $\left(T_{\text {н.р. } j}\right)$ при разогреве плавильной печи; $\tau_{2, j}$ - время достижения температуры $T_{\text {н.р. }}$ при охлаждении разогретой реакционной сре- ды. Очевидно, что $\tau_{1, j}$ и $\tau_{2, j}$ есть функции координат.

Данные по выходу газообразных продуктов при производстве карбида кремния для разных составов шихты известны. Эти данные получают при проведении соответствующих замеров на практике для контроля за исполнением экологических требований по выбросам побочных продуктов производства в окружающую среду. Обычно эти данные представлены как выход газообразного продукта реакции «ј» на единицу массы $\left(\mathrm{M}_{\text {загр }}\right)$ загружаемой шихты, $G_{\text {хим, }, j}^{\text {э }}$.

Тогда, в рамках принятой модели,

$\widetilde{g}_{\text {хим }, j}=\frac{G_{\text {хим }, j}^{\text {э }} \mathrm{M}_{\text {загр }}}{\int_{V \mathrm{p}, j}\left(\tau_{2, j}-\tau_{1, j}\right) d V}$,

где подинтегральные функции $\tau_{1, j}$ и $\tau_{2, j}$ определяются с использованием экспериментальных данных по температурным полям по производству карбида кремния [1].

\section{Определение $q_{V, \text { суш }} \cdot$}

В работе [1] представлена модель сушки пористого материала при внутреннем нагреве. Поскольку эта модель сушки полностью адекватна общей модели процессов, принятой в настоящей работе, $\widetilde{g}_{\text {суш }}$ определяется согласно модели [1], а $q_{V \text {,суш }}$ по соотношению (6).

определение $q_{V, \phi}$.

В работах [2] и [3] описана и верифицирована модель расчёта поля давления в пористой среде с реагирующими компонентами применительно к процессу производства карбида кремния.

При известном распределении давления фильтрационная составляющая $\tilde{g}_{\phi}$ определяется по модели Дарси, а $q_{V, \phi}$ по соотношению (8).

\section{Модель расчёта эффективного коэффициента теплопроводности $\lambda_{\text {эф }}$}

Рассмотрим модель по определению эффективного коэффициента теплопроводности двухкомпонентной системы $\lambda_{\text {эф }}$, фактически фигурирующего в опеpaтope $\operatorname{div} q$ правой части базового уравнения (1) и (9):

$$
\operatorname{div} q=-\operatorname{div}\left(\lambda_{э ф} \operatorname{grad} T\right) .
$$

Пусть в частном варианте «а» двухкомпонентная система имеет вид чередующихся слоев однородных компонентов, когда можно считать, что тепловой поток направлен вдоль слоев. Пусть, как принято ранее, $\varepsilon$ - объёмная доля компонента «2». 
Тогда в рамках модели сплошной среды

$\lambda_{э ф, \mathrm{a}}=\lambda_{1}(1-\varepsilon)+\lambda_{2} \varepsilon$

где «а»-индекс варианта.

Пусть в ином частном варианте «б» двухкомпонентная система имеет вид чередующихся слоев однородных компонентов, когда можно считать, что тепловой поток направлен поперек слоев по нормали к ним. Тогда

$\lambda_{\text {эф, }}=\left(\frac{1-\varepsilon}{\lambda_{1}}+\frac{\varepsilon}{\lambda_{2}}\right)^{-1}$,

где «б» индекс варианта.

Полагая, что в пористой системе многообразная форма пор и их случайное расположение определяют равную вероятность любого угла $\varphi\left(0<\varphi<90^{\circ}\right)$ между нормалью к поверхности раздела «твердая составляющая-газовая составляющая» и вектором плотности теплового потока, имеем из (17) и (18):

$\lambda_{\text {эф }}=\frac{2 \lambda_{1} \lambda_{2}+\varepsilon(1-\varepsilon)\left(\lambda_{1}-\lambda_{2}\right)^{2}}{2\left[\lambda_{2}(1-\varepsilon)+\lambda_{1} \varepsilon\right]}$.

\section{Модель изменения пористости $\varepsilon$ системы}

По определению

$$
\begin{aligned}
& \tilde{g}_{\text {хим }}=-\frac{\partial \tilde{\rho}_{1}^{c}}{\partial \tau}=-\frac{1}{1+v}\left\{(1-\varepsilon) \frac{\partial \rho_{1}^{c}}{\partial \tau}-\rho_{1}^{c} \frac{\partial \varepsilon}{\partial \tau}\right\} . \text { Откуда } \\
& \frac{\partial \varepsilon}{\partial \tau}=\frac{1}{\rho_{1}^{c}}\left\{(1+v) \tilde{g}_{\text {хим }}+(1-\varepsilon) \frac{\partial \rho_{1}^{c}}{\partial \tau}\right\} .
\end{aligned}
$$

И изменяющаяся во времени пористость системы будет определена как $\varepsilon=\varepsilon_{0}+\int_{\tau} \frac{\partial \varepsilon}{\partial \tau} d \tau$.

Заметим, что производная $\frac{\partial \rho_{1}^{c}}{\partial \tau}$ в соотношении (20) может быть представлена как $\frac{\partial \rho_{1}^{c}}{\partial T} \frac{\partial T}{\partial \tau} \quad$ с определением $\frac{\partial \rho_{1}^{\mathrm{c}}}{\partial T}$ по справочным данным для материалов шихты.

\section{Выводы}

Разработка модели тепломассопереноса в печах при производстве $\mathrm{SiC}$ потребовала подробного анализа физических процессов тепло- и массобмена в процессе плавки карбида кремния, с принятием во внимание большого числа теплофизических параметров. В модели учтены основные факторы, влияющие на интенсивность процессов тепломассообмена в электропечах сопротивления, а именно: теплота химических реакций, образование дополнительной газовой составляющей, процесс сушки, перенос тепла фильтрационным потоком газа. Математически описан тепломассобмен в пористой среде с внутренними источниками тепла. Полученная математическая модель позволяет определить температурные поля в процессе образования карборунда и подобрать варианты загрузок печи и режимов плавки карбида кремния с целью увеличения выхода продукта и экономии энергоресурсов.

\section{Список литературы}

1. Кузеванов В.С., Закожурникова Г.С. Модель сушки пористого проницаемого материала при внутреннем нагреве // Международный научный журнал «Альтернативная энергетика и экология» (ISJAEE). 2013. № 14. C. 19-23.

2. Кузеванов В.С., Закожурникова Г.С. Общая модель для расчета поля давления в пористой среде с реагирующими компонентами // Известия ВолгГТУ. Серия «Процессы преобразования энергии и энергетические установки». 2014. Т. 18, № 6 (145). С. 106-110.

3. Кузеванов В.С., Закожурникова Г.С. Расчет поля давления в пористой среде с реагирующими компонентами // Известия ВолгГТУ. Серия «Процессы преобразования энергии и энергетические установки». 2014. Т. 18, № 6 (145). С. 110-113.

\section{References}

1. Kuzevanov V.S., Zakozhurnikova G.S. Model' suški poristogo pronicaemogo materiala pri vnutrennem nagreve. International Scientific Journal "Al'ternativnâ̂ ènergetika i èkologiâ” (ISJAEE), 2013, no. 14, pp. 19-23.

2. Kuzevanov V.S., Zakozhurnikova G.S. Obŝaâ model' dlâ rasčeta polâ davleniâ v po-ristoj srede $\mathrm{s}$ reagiruûsimimi komponentami. Izvestiâ VolgGTU. Seriâ "Processy pre-obrazovaniâ ènergii i ènergetičeskie ustanovki”, 2014, vol. 18, no. 6 (145), pp. 106-110.

3. Kuzevanov V.S., Zakozhurnikova G.S. Rasčet polâ davleniâ v poristoj srede s reagi-ruûsiimi komponentami. Izvestiâ VolgGTU. Seriâ "Processy preobrazovaniâ ènergii i ènergetičeskie ustanovki”, 2014, vol. 18, no. 6 (145), pp. 110-113.

Транслитерацчия по ISO 9:1995 